\title{
Plug and Play Networking with Optical Nodes
}

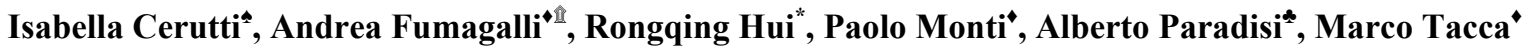 \\ ${ }^{*}$ CEIRC research center, Scuola Superiore Sant'Anna, 56124, Pisa, Italy \\ * Department of EECS, The University of Kansas, Lawrence KS, 66044, USA \\ *Networking Systems Development, CPqD, Rodovia Campinas-Mogi Mirim km.118, Campinas (SP), Brazil \\ - OpNeAR Lab, University of Texas at Dallas (UTD), Richardson, TX, 75083, USA \\ ${ }^{\mathbb{1} T e l:}+1$ (972) 883 6853,Fax: +1 (972)883 2710,e-mail: andreaf@utdallas.edu
}

\begin{abstract}
Plug and play optical (PPO) nodes may be used to facilitate the deployment of optical networks. PPO nodes must be able to learn about the signal propagation properties of the surrounding optical fibers and make their wavelength routing decisions based on the collected data. This paper discusses what are the open challenges that must be overcome to provide cost effective and performing ad hoc networking solutions based on PPO nodes.

Keywords: plug and play networking, plug and play optical nodes, ad-hoc access, on-board optical mini-lab, physical impairments, optical networking.
\end{abstract}

\section{INTRODUCTION}

The latest advances in optics widely demonstrate that today's technologies have the potential to enable end-toend user communications in the gigabit transmission range [1]. Some of the advantages of optical networking include the possibility to reduce the electronic processing within the network and to benefit from the degree of transparency [2] provided by setting up optical circuits, or lightpaths [3], in wavelength routed networks. However, the deployment of optical networks is mainly delayed by two factors: the excessive cost of optical nodes and the highly complex procedures for the design, installation, and maintenance of the overall network.

The high cost of an optical node is partially due to low scale fabrication and lack of integrated solutions at this time. The network design complexity originates from the fact that a large number of measurements must be performed in the laboratory to make sure that the network planning phase has been correctly performed by trained engineers. These measurements mainly relate to the quality of the optical signals, e.g., optical signal-tonoise ratio (OSNR), while propagating across the fibers and the optical nodes. Notice that in conventional (first generation) optical networks the signal propagation is limited within two physically adjacent nodes, which greatly limits the number of measurements that need to be performed. The network installation is complex, because upon equipment installation, several on-field measurements are required to confirm the proper installation of the equipment. Network management is complex too, since continuous monitoring of the optical signal quality must be performed to detect malfunctions, and, where possible, to anticipate element failures. In addition to the above burdens, whenever an upgrade is needed in the network, the entire process must be repeated, as newly added nodes affect existing nodes and the way optical circuits are established.

To circumvent the cost and complexity burden of extant optical networks, the Authors propose to investigate an unconventional approach to designing ad-hoc optical networks that may become viable over the next decade or so. The enabling component of the proposed approach is a cost-effective, self-configurable Plug-and-Play Optical (PPO) node.

PPO nodes consists of an optical mux/demux and switch fabric for optical circuit switching, a miniature optical transmission laboratory (mini-lab) module for signal quality monitoring and processing, and lower speed service channel interfaces for network management and control. Its cost is contained by: 1) avoiding electronic processing of user data packets at the PPO node, thanks to the so called optical transparency [2], [3]; 2) using low speed service channels between PPO nodes and user interfaces; and 3) including the mini-lab for optical signal monitoring and performance assessment. In the mini-lab, simple real-time transmission models process the measurements produced and provide estimation of the transmission rates allowed between clients - e.g., endusers, routers, and other electronic nodes.

Similar to today's Fast Ethernet switches capabilities, ad-hoc optical topology could be easily deployed by connecting a large number of PPO nodes, via already existing fibers. Once connected, a PPO node will cooperate with the already existing nodes, and will efficiently make use of available optical resources, such as fibers and wavelengths, to provide high speed optical circuits to the connected clients.

An example of the envisioned ad-hoc network is shown in Fig. 1. In the figure, only a subset of PPO nodes and I/O ports are connected to electronic client nodes. A user can request the creation of a lightpath for transmitting data directly to another user (single-hop transmission). Alternatively, a user can request a lightpath to reach a preferred router. With this option, the router will perform electronic forwarding of the received packets towards the intended final destinations (multi-hop transmission). Additionally, the router can statistically

The work reported in this paper was supported in part by NSF (Grants No. ANI-0435393 and ANI-0435381) and the Italian Ministry of University (MIUR) (contract \# RBNEO1KNFP). 
multiplex multiple user connection requests on a single lightpath. Routers can also request lightpaths to be created to reach other routers or desired end-users. With any of these lightpath creation alternatives, a technique similar to the one used by modem technology is envisioned, in which a lightpath (the equivalent of the phone circuit) is requested, and the transmission rate is selected based on the channel quality. Contrary to the user determining the phone number to call, in the envisioned PPO scenario a distributed and automatic procedure determines: 1) which client must connect to which client and 2) which route, wavelength, and transmission rate ought to be used to achieve good performance, e.g., minimize network congestion and blocking probability of user connection requests. Due to the large number of possible circuits provided by the PPO nodes and the numerous phenomena affecting the quality of the optical circuits, e.g., chromatic dispersion and polarization mode dispersion (PMD), to mention a few, the resulting optimization problem is much more complex than the one in the case of modem technology.

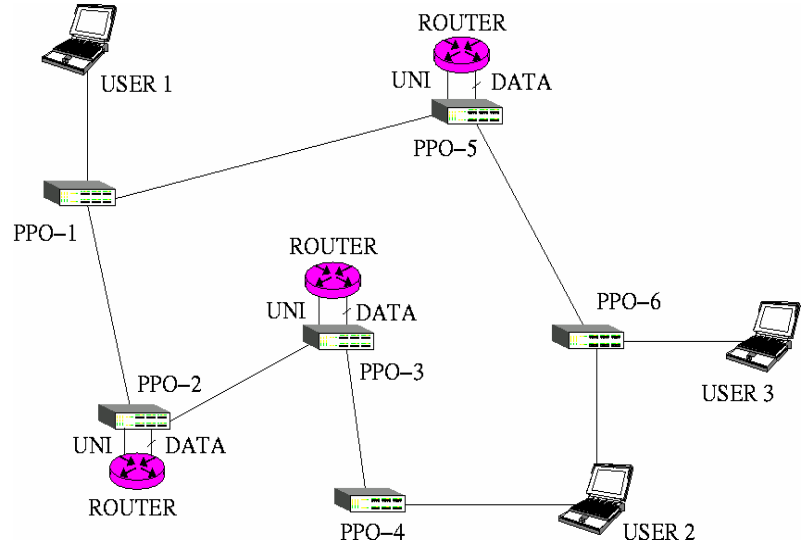

Figure 1. PPO node and network concept.

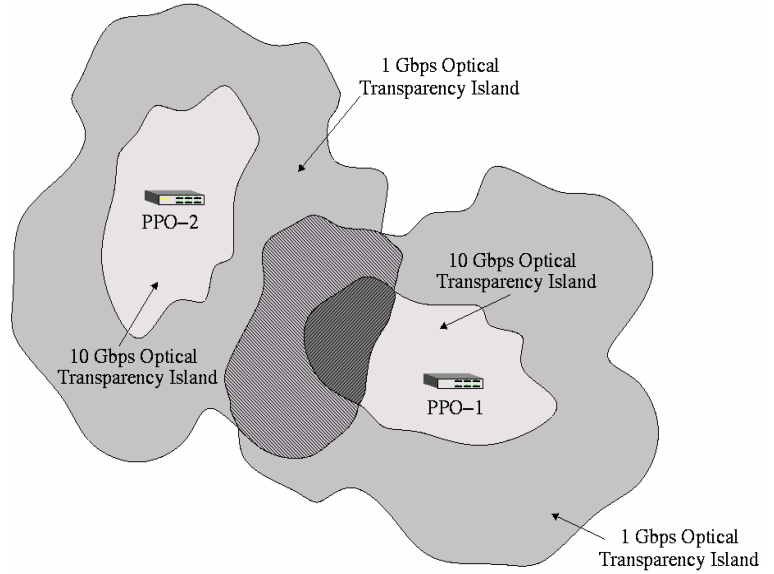

Figure 2. Optical transparency island.

Once the above problem is solved, the design, installation and management of the proposed ad-hoc optical network will be extremely simple due to the fact that PPO nodes have built-in capabilities for auto-discovery, self-configuration, and self-monitoring. There is no need for complex network planning, no engineering team is required in the field to check correct installation of equipment and to monitor network functions. These tasks will be automatically performed by the PPO nodes in a cooperative manner, by using the measured network parameters and the on-board signal processing features. As a result, minimal training will be required to install, operate, and maintain such nodes, thus considerably reducing the overall network cost. A large number of PPO nodes can thus be deployed to create ad-hoc access, local area, metropolitan area, and possibly wide area networks that self maintain, configure, protect, and recover from failures with minimal human intervention.

Among the applications that will benefit from the availability of PPO nodes, one can include rapidly deployable wide-bandwidth sensor networks, in which each sensor is expected to generate multi-gigabit data streams [4][5]. The PPO node self-configuring capability will enable rapid deployment and installation of both PPO nodes and wide bandwidth sensors, without requiring experienced engineers in optical networking.

\section{FURTHER DESCRIPTION OF PPO NODE AND NETWORK FUNCTIONALITIES}

This section provides a more detailed description of the proposed PPO node and network concept. The envisioned PPO network architecture is based on the assumption that in few years from now, gigabit Ethernet cards will be inexpensive, following the same cost pattern of today's Fast Ethernet. Thus, end-users will have direct access to wavelength channels. These gigabit cards are expected to have tunable capabilities (in transmission and reception), variable bit rate (multi-rate) interfaces, adjustable transmission power, and a dedicated lower rate service channel, used for signaling between the network ingress point (the PPO node which is physically connected to the user card) and the user card. Similar features are expected to be available at the interfaces of the routers that are connected to PPO nodes. End-users, routers, and other electronic nodes are generally referred to as clients in this section, as PPO nodes and the optical layer act as a server for them.

Upon request, PPO nodes must provide clients with lightpaths that may span across multiple PPO nodes. Due to various transmission impairments and network status, it might not be always possible to connect two clients using a lightpath at the desired transmission rate. For this reason, the PPO node must first be able to use accurate models of the transmission impairments in order to predict the quality of any given lightpath [6]. In addition, models must take into account the effects that lightpaths sharing the same fiber, on spectrally adjacent wavelengths, may have on the optical signal quality. 
An exhaustive trial-and-error approach is not practical due to the number of possible lightpath alternatives that grows exponentially with the number of PPO nodes, client nodes and wavelengths. Predicted transmission rates must be verified once the lightpath is created using the mini-lab available at the PPO nodes. The result of this transmission analysis indicates which clients can be connected, and which transmission rates can be used without significant signal degradation of the lightpath under consideration and of the spectrally adjacent lightpaths.

It is possible to define the optical transparency island [7], [8] of a given PPO node as the subset of PPO nodes that can be reached all-optically from that PPO node. Notice that an optical transparency island is individually defined for each PPO node, and may partially overlap with other PPO nodes' islands. Its extension is strongly dependent on its transmission rate, as illustrated in Fig. 2, and might vary over time due to changes of physical parameters (e.g., temperature) and distribution of the existing lightpaths. The PPO node can detect and react to such changes by using and processing the measurements produced by the on-board mini-lab.

Whenever two clients cannot be connected using a single lightpath due to transmission impairments, enduser connection requests are fulfilled using a concatenation of routers and lightpaths using multi-hop transmission. To accomplish these various tasks, three modules are required:

- the PPO network (server layer) management and control module;

- the client network management and control module;

- the on-board miniature optical transmission (mini-lab) module.

\subsection{PPO Network Management and Control Module}

This module is responsible for:

1. setting up, tearing down, and reconfiguring lightpaths to satisfy client requests;

2. advertising and auto-discovering of available resources within the optical transparency island;

3. predicting the effect of transmission impairments using analytical models, e.g., determining if a lightpath is feasible, at what transmission rate, and the impact of a newly created lightpath on already existing lightpaths;

4. monitoring the status of the network, e.g., detecting optical signal degradation of existing lightpaths, possibly malfunctioning client interfaces, changes of transmission parameters.

PPO nodes can use a service channel on a dedicated control wavelength (e.g., at $1.3 \mu \mathrm{m}$ outside the amplifier window) to exchange control information with physically adjacent PPO nodes. For cost-effectiveness, the transmission rate of the service channel may be limited (compared to data channels), e.g., $100 \mathrm{Mbps}$. Due to the ad-hoc nature of the PPO network, scalability is of paramount importance. Scalability is achieved using the PPO node optical transparency island. The management and control protocol constraints the exchange of control messages (e.g., in the form of IP packets) generated by a PPO node within its optical transparency island. Notice that although the PPO node optical transparency island is similar to the concept of autonomous system in IP networks, it has significant differences: it may change over time, it is automatically determined by the PPO node, and each PPO node has its own optical transparency island.

By exchanging control messages, each PPO node maintains two distinct databases. The first database is used to keep track of available resources within the optical transparency island of the PPO node and to self-discover newly added/removed resources. The second database keeps track of both the measurements made by the minilab - both locally and at remote PPO nodes - and of the information required and generated by the transmission models. Both databases can be updated using extensions of the OSPF-TE protocol [9]. In certain instances, it may be necessary to create temporary dummy lightpaths between PPO nodes to test transmission impairments, e.g., generate the dispersion map, and provide more accurate estimates of the optical signal quality.

A client communicates with its adjacent PPO node(s) via a User to Network Interface (UNI) using the service channel. A client requests the creation of a lightpath using the UNI. Upon receiving a request for a lightpath, the PPO node solves the routing and wavelength assignment (RWA) problem [8], [10], [11] by using both databases. In addition to solving the conventional RWA problem, the PPO node must determine a set of physical parameters that characterize the profile of the requested lightpath, e.g., range of acceptable transmitted power, transmission rate, maximum acceptable wavelength drift, etc. Transmission models are used by the PPO node to ensure that the profile of the newly created lightpath conforms to the client request, without negatively impacting the already created lightpaths in the network. During the lifetime of the lightpath, the PPO node continuously monitors the signal received from the client to make sure that it meets the assigned profile. In the case of violation, the PPO node could, for example, send a disconnect message and release the network resources to other lightpath requests.

\subsection{Client Network Management and Control Module}

This module is responsible for handling end-to-end traffic flows at the electronic layer. Transmission impairments limit the reach of a PPO node, i.e., the size of its optical transparency island. In order to provide 
clients with connectivity that goes beyond the optical transparency island, multi-hop transmission must be used. In this case, the end-user data traffic is electronically processed by a cascade of routers.

Routers handle client packets electronically, and can operate at a much finer multiplexing granularity than the wavelength granularity of a lightpath. For example, MPLS [12], [13] can be used to provide traffic engineering at this layer. Routers have a set of input ports which are equipped with Optical Line Terminals (OLT) that convert the incoming optical signal into the electronic domain. Output ports are equipped with tunable lasers that convert electronic signals back into the optical domain.

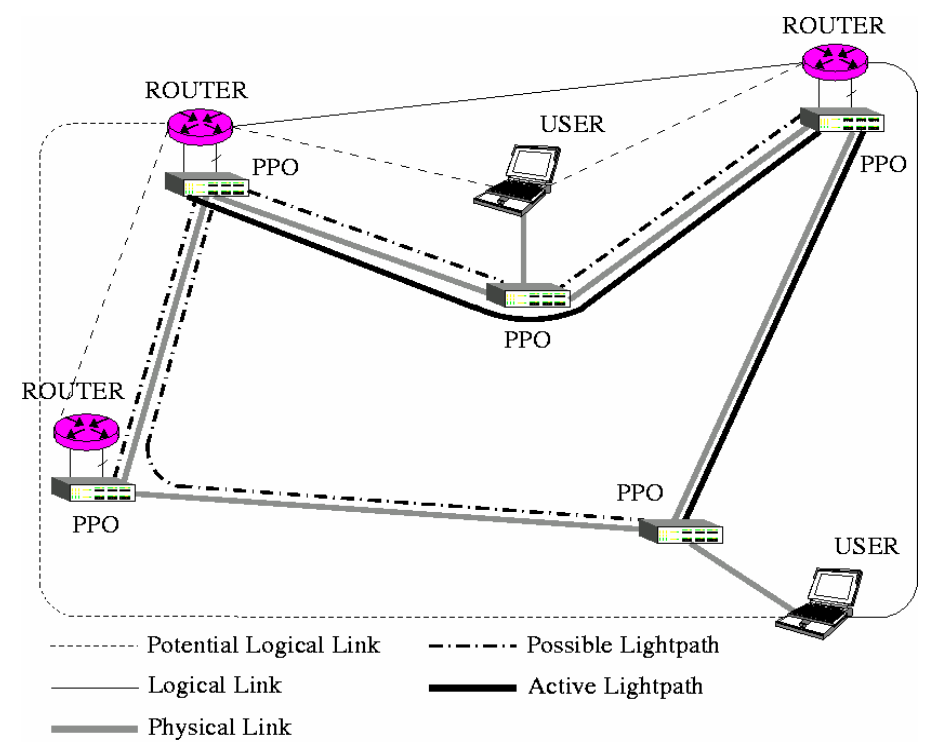

Figure 3. Logical links and potential logical links.

At the electronic layer, control messages are exchanged using inband signaling, i.e., routers exchange control messages using the available logical links ${ }^{1}$. Being clients, routers communicate with the underlying PPO node through the UNI. Using both the service channel and inband signaling, each router maintains two routing tables. The first one (T1) keeps track of the reserved resources, using extensions of standard IGP protocols [14], such as OSPF-TE. The second routing table (T2) keeps track of the potential logical links (lightpaths) that could be created by the PPO nodes (Fig. 3) - in case the router requests the underlying PPO node to set up a new lightpath using the UNI. T2 may be updated using two different techniques. The first technique makes use of the UNI toward the PPO node, which provides to the router the list of routers that can be reached within the optical transparency island. The information collected this way is then advertised to other routers using inband signaling over existing lightpaths. The second technique makes use of the dummy lightpaths that are created periodically by the PPO nodes to measure the transmission parameters of the optical layer. While dummy lightpaths are created, the router can exchange control messages with the routers at the other end of the dummy lightpaths.

With the described PPO node network architecture, a client node, say router R1, has different options to choose from when it attempts to establish a data flow with another client: use already existing lightpaths, i.e., using routing table $\mathrm{T} 1$ to reach the destination, or create new lightpath(s) using routing table $\mathrm{T} 2$. In the latter case, R1 requests that the underlying PPO node creates a lightpath to reach the intended destination client. If the PPO node determines that the required connection is possible within its optical transparency island, the new lightpath is created. If such lightpath is not possible, or the allowed transmission rate on such lightpath is not sufficient, R1 must select an intermediate router, say R2, which is within reach of the PPO optical transparency island. $\mathrm{R} 2$ repeats the same procedure as $\mathrm{R} 1$. The multi-hopping procedure is repeated until the client destination node is reached.

With the above routing strategies, routers are provided with unconventional techniques to handle congestion, in addition to the conventional congestion avoidance procedure, e.g., Random Early Discard (RED) strategy. For example, the router can operate locally with the underlying PPO node using the UNI, and request additional bandwidth on the outgoing logical links ${ }^{2}$. Another alternative is to reduce the traffic coming from upstream neighbouring routers, using the inband signaling mechanism, and requesting them to create new lightpaths that bypass the congested router.

\footnotetext{
${ }^{1}$ Lightpaths in the optical layer correspond to logical links at the electronic layer.

2 The PPO node can fulfill this request by either increasing, if possible, the transmission rate of the outgoing lightpaths or by creating additional lightpaths.
} 


\subsection{On-board Miniature Optical Transmission Laboratory (Mini-Lab) Module}

A miniature optical transmission laboratory (or mini-lab) module is required at each PPO node. With the help of the mini-lab module, the PPO node can produce and frequently update the information about the optical physical layer, which is required by the PPO network management and control module. The mini-lab module accomplishes a number of key monitoring tasks. For example, one task is to provide physical layer information of the optical network elements which will provide important guidelines while setting up lightpaths. Another task is to monitor the performance of the optical network after lightpaths are set up and the network is in operation. The produced performance information is used by the PPO network management and control module to optimize and adjust traffic and lightpaths. The mini-lab module requires simplicity of configuration and easy integration of its components. On the other hand, in order to self-determine the network connectivity and transmission characteristics, this module needs to provide sufficient functionalities at a limited cost. An example of coherent detection PPO node is described next.

Assume that two neighbouring PPO nodes have agreed to characterize their connecting fiber pair. Each PPO node makes use of a single tunable laser diode to determine chromatic dispersion, nonlinearity and PMD of the fiber pair, as follows. At one PPO node the tunable laser is used as transmitter. At the other PPO node the tunable laser is used as the local oscillator for coherent detection. Physical layer information acquired via coherent detection (examples can be found in [15]) is then fed into the transmission models for network performance evaluation and prediction, which provide meaningful information to the PPO network management and control module.

At the PPO node, each input fiber port has a low cost and low speed receiver. These receivers monitor the optical signal connectivity between PPO nodes. To find additional fiber system information, a linearly swept RF source is used to drive the modulator. The frequency response of the fiber system can then be measured by the coherent detection optical receiver. Synchronization is not required in this measurement as the modulation frequency can be found at the receiver by evaluating the modulation sidebands with the local oscillator performing coherent spectrum analysis. When the optical signal power is small enough, the frequency response of the fiber system is a function of the accumulated chromatic dispersion. Therefore the dispersion of the fiber system can be precisely evaluated by this measurement. By increasing the signal optical power, the frequency response of the fiber system will be modified compared to the linear condition and this change depends on the nonlinear parameter of the fiber used in the system. The measurement of the dependency of the fiber system frequency response on the optical signal power can then be used to evaluate the nonlinearity of the fiber system [15]. With both linear and nonlinear parameters of the system being measured, system modeling can be performed to predict the link performance at various data rates. This information is essential for the successful setup of lightpaths.

When lightpaths are set up and data traffic is being carried, the same coherent detection monitoring module may be used to perform in-situ monitoring of optical link performance such as the variation of OSNR and PMD. In particular, to evaluate PMD the coherent detection receiver in the mini-lab module down converts the optical spectrum of the data channel in the RF domain. Two different frequency components are selected by bandpass RF filters and their relative polarization walk-off can be analyzed, which predicts the differential group delay (DGD) of the fiber system [16].

\section{CONCLUSION}

Several are the open challenges in pursuing the described PPO node concept. Because of the optical transparency, existing solutions that are successful in conventional data networks (SONET, ATM, Ethernet) are not suitable in this scenario. Self-handling of optical transmission impairment requires innovative approaches and protocols that cannot be found in today's commercial solutions.

Another challenge is represented by the fact that all-optical networks have been studied for more than a decade, and yet commercially viable solutions have not been found. Although optical/RF signal processing methods to predict and monitoring optical link performance have been proposed and demonstrated, optoelectronic integration will be necessary in the future to realize a true low-cost and miniaturized plug-and-play module that may pave the way to solutions that have been unthinkable thus far.

If proven feasible, the proposed architecture based on PPO nodes will provide a self-configuring solution that continuously determines a cost effective way to employ (optical) network resources throughout the lifetime of the network, i.e., 1) it determines the most cost effective solution by using all-optical transmission and/or $\mathrm{O} / \mathrm{E} / \mathrm{O}$ (through routers) at selected nodes on a per flow basis; 2) it provides dynamic bandwidth provisioning that adjusts to traffic changes, i.e., as traffic patterns change, the network can set up new lightpaths, and tear down old ones, thus avoiding the problem of burning wavelengths (fixed reservation of wavelengths); 3 ) as new PPO nodes and fibers are added to the network, it discovers them automatically, without requiring the manual redesign of the network, similarly to today's "plug-and-play" network nodes in LAN technology; 4) it provides timely monitoring of the signal quality on each optical circuit to inform the network management and control 
module; 5) it provides an optical and RF signal processing method to predict and monitor the performance of the optical circuits in the network.

The self-configuration capability of the architecture based on PPO nodes makes it possible to reduce the network design, installation, and maintenance costs, because no-human intervention is required to perform complex tasks. The success of the deployment of self-configuring networks highly depends also on the possibility of developing high performance, compact, and easy-to-install PPO nodes.

In conclusion, it is interesting to notice that some similarities between the proposed ad-hoc optical networks and the dynamic nature of ad-hoc wireless networks can be found here. New PPO nodes can be deployed by the user as needed, and connected to existing fiber cables. A user interface may migrate physically from one PPO node to another. A user interface may migrate logically from one router to another, i.e., creating a new adjacency in the routing tables, by simply requesting a new lightpath. All these unknowns, combined with the complexity of handling optical signals quality at high transmission rates, constitute a set of challenging open problems that must be tackled.

\section{ACKNOWLEDGEMENTS}

The authors would like to thank Prof. Stefano Gregori and Prof. Franco Maloberti for their valuable technical conversations and inputs.

\section{REFERENCES}

[1] N. M. Froberg, et al., "The NGI ONRAMP test bed: reconfigurable WDM technology for next generation regional access networks," Journal of Lightwave Technology, vol. 18, no. 12, pp. 1697-1708, December 2000 .

[2] C. A. Brackett: Dense wavelength division networks: principles and applications, IEEE Journal on Selected Areas in Communications, vol. 8, pp. 373-380, August 1989.

[3] I. Chlamtac, A. Ganz and G. Karmi, "Lightpath communications: a novel approach to high bandwidth optical WAN's," IEEE Transactions on Communication, vol. 40, no. 7, pp. 1171-1182, July 1992.

[4] S. L. Bernstein, et al.: Wideband networked sensors, in Next Generation Internet Principal Investigator Meeting, www.fas.org/spp/military/program/track/martinez.pdf, October 2002.

[5] R. Fontana, "Current trends in UWB systems in the USA implementation, applications and regulatory issues," in Advanced Radio Technology Symposium 2002, www.multispectral.com/pdf/MSSI $\backslash$ ARTS.pdf, 2002.

[6] J. Strand, A. Chiu, "Impairments and other constraints on optical layer routing," in RFC 4054, May 2000.

[7] A. A.M. Saleh, "Islands of transparency - an emerging reality in multiwavelength optical networking," in IEEE/LEOS summer topical meeting on broadband optical networks and technologies, Monterey, CA, July 1998.

[8] P. E. Green, "Fiber optic networks," Englewood Cliffs (NJ): Prentice-Hall, 1993.

[9] S. Das, et al., "A link state advertisement (LSA) protocol for optical transparency islands," The University of Texas at Dallas, Technical Report UTD/EE/02/2006, http://opnear.utdallas.edu, April 2006.

[10] B. Mukherjee, “Optical communications networks," S. M. Elliot Ed., McGraw Hill Ed., 1987.

[11] R. Ramaswami and K. N. Sivarajan, "Optical networks: a practical perspective (Second Edition)," R. Adams Ed., Morgan Kaufmann Publishers, Inc., 2002.

[12] A. Banerjee, et al., "Generalized multiprotocol label switching: an overview of signaling enhancements and recovery techniques," IEEE Communications Magazine, vol. 39, no. 7, pp. 144-150, July 2001.

[13] D. Awduche, Y. Rekhter, "Multiprotocol lambda switching: combining MPLS traffic engineering control with optical crossconnects," IEEE Communications Magazine, vol.39, no .3, pp. 111-116, March 2001.

[14] R.W. Stevens, “TCP/IP illustrated, volume 1,” Addison-Wesley Professional Computing Series, 1994.

[15] R. Hui, A. Fumagalli, "Plug-and-play optical node architectures and their built-in optical fiber characterization techniques," The University of Texas at Dallas, Technical Report UTD/EE/03/2006, http://opnear.utdallas.edu, May 2006.

[16] B. Fu, R. Hui, "Fiber chromatic dispersion and polarization-mode dispersion monitoring using coherent detection," IEEE Photonics Technology Letters, vol. 17, no. 7, pp. 1561-1563, July 2005. 